\title{
Heimlich maneuver to relieve choking caused by a duodenal subepithelial tumor that was resected by endoscopic mucosal resection
}

We present a rare case of choking caused by a duodenal subepithelial tumor resected by endoscopic mucosal resection (EMR). The Heimlich maneuver was performed successfully to relieve the patient's choking.

A 56-year-old man was found to have a large subepithelial tumor at the duodenal bulb ( Fig.1), with the initial presentation of postprandial epigastric fullness. Endoscopic ultrasonography (EUS) (Olympus UM-3R, 20-MHz radial miniprobe; Olympus, Tokyo, Japan) revealed a $21.4 \times$ $18.0 \mathrm{~mm}$ inhomogeneous isoechoic tumor with a cystic component $(8.9 \times 6.2 \mathrm{~mm})$ inside, arising from the submucosal layer of duodenal wall ( $\bullet$ Fig. 2). A Brunner's gland adenoma was suspected; however, the possibility of malignancy could not be excluded by EUS [1].

EMR was performed for both therapeutic and diagnostic purposes. The endoscopic overtube (MD-48518; Sumitomo Bakelite Co. Ltd., Tokyo, Japan), which had an inner diameter of $15 \mathrm{~mm}$, was not used, because the tumor might have been too large to be taken out through it. With the patient under intravenous conscious sedation (Rapifen, $0.5 \mathrm{mg}$; midazolam, $3 \mathrm{mg}$; propofol, $400 \mathrm{mg}$ in total), the tumor was resected with a snare (Olympus SD-9U-1) after submucosal injection with saline. The resected tumor was partially captured by a retrieval net (Roth Net foreign body standard; US Endoscopy, Mentor, Ohio, USA). However, the specimen fell out in the hypopharynx and was sucked into the trachea ( $\bullet$ Video 1 ).

We moved the patient from the left decubitus position to a supine position and performed the Heimlich maneuver by compressing the patient's epigastric area

\section{Video 1}

Removal of a resected duodenal subepithelial tumor. The tumor was partially captured by a retrieval net, but the specimen fell out in the hypopharynx and was sucked into the trachea. with our fists. Immediately, the specimen went back into the oral cavity, and a foreign body removal forceps (rotatable grasping forceps; Olympus) was used to take it out ( $\bullet$ Fig.3). Histopathological analysis confirmed the diagnosis of Brunner's gland adenoma. The patient recovered well without any complications.

EMR was performed in this case mainly to relieve the patient's partial gastric outlet obstruction caused by the tumor. Endoscopic removal of a large Brunner's gland adenoma has been suggested to prevent hemorrhage, obstruction, or intussusception [2]. In addition, the diagnostic accuracy of EUS in subepithelial tumors is low [1]. A malignant tumor can sometimes mimic a benign subepithelial tumor [3]. Moreover, although most Brunner's gland adenomas are benign tumors, they still have malignant potential [4].

When the airway is obstructed, laryngoscopy or bronchoscopy can be applied to remove the foreign body. However, because both procedures are time consuming, urgent trachea intubation is required first to avoid asphyxia. Intubation may push the specimen to a deeper part of the trachea. Thus we did the Heimlich maneuver to relieve the patient's airway obstruction. The Heimlich maneuver is quite useful to remove a foreign body in the airway in patients who are fully conscious and in a standing position [5]. To our knowledge, this is the first case that reports use of the Heimlich maneuver in a sedated patient during EMR.

In EMR, airway obstruction by a resected specimen is rare. The Heimlich maneuver allows the missing specimen to be obtained instantly and the patient to be saved.

Endoscopy_UCTN_Code_CPL_1AH_2AJ

Competing interests: None

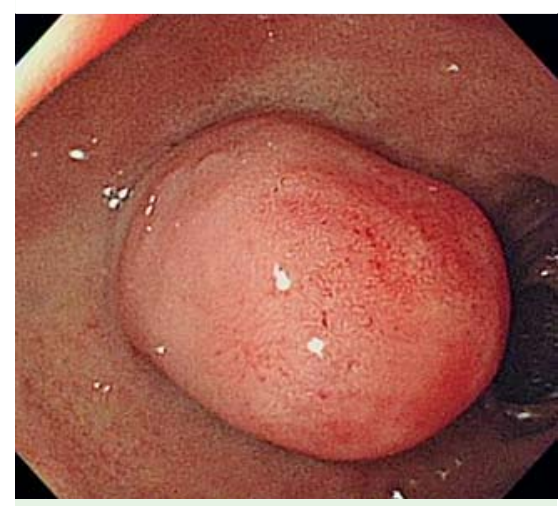

Fig. 1 A large protruding tumor at the anterior wall of duodenal bulb.

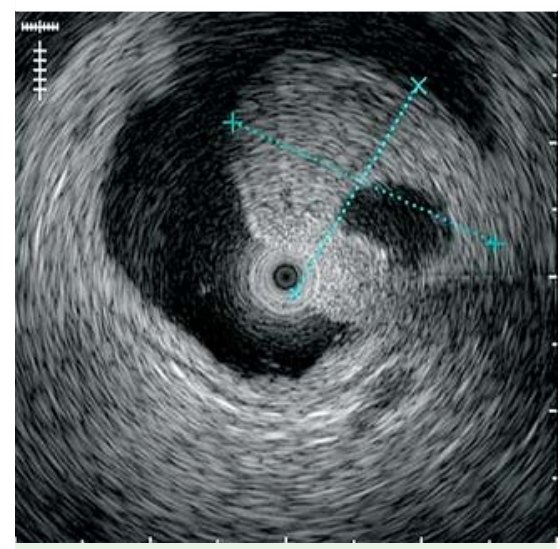

Fig. 2 The submucosal tumor $(21.4 \times 18.0 \mathrm{~mm})$ (shown by two dashed lines) with a cystic component inside.

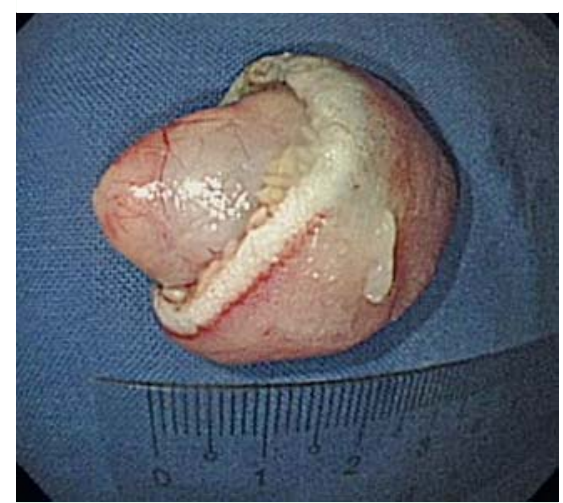

Fig. 3 The retrieved resected specimen. The final diagnosis was Brunner's gland adenoma. 
W.-H. Liao ${ }^{1}$, C.-S. Chen ${ }^{2}$, P.-F. Liu' ${ }^{1}$, W.-H. Lee ${ }^{3}$, I.-L. Lee ${ }^{1}$

${ }^{1}$ Division of Gastroenterology, Department of Internal Medicine, Shuang Ho Hospital, Taipei Medical University, New Taipei City, Taiwan

2 Department of Anesthesiology, Shuang Ho Hospital, Taipei Medical University, New Taipei City, Taiwan

${ }^{3}$ Department of Pathology, Shuang Ho Hospital, Taipei Medical University, New Taipei City, Taiwan

\section{References}

1 Sakamoto H, Kitano M, Kudo M et al. Diagnosis of subepithelial tumors in the upper gastrointestinal tract by endoscopic ultrasonography. World J Radiol 2010; 28: 289 297

2 Rocco A, Borriello P, Compare D et al. Large Brunner's gland adenoma: Case report and literature review. World J Gastroenterol 2006; 28: 1966 - 1968

3 Kojima Y, Kobayashi T, Note M et al. A case of duodenal carcinoma presenting as a submucosal tumor. Surg Today 1992; 22: 357-362

4 Fujimaki E, Nakamura S, Sugai T et al. Brunner's gland adenoma with a focus of p53positive atypical glands. J Gastroenterol 2000; 35: 155-158

5 Arash S, Holly ES, Barbara MS et al. Adult foreign body airway obstruction in the prehospital setting. Prehosp Emerg Care 2007; 1: $25-29$

\section{Bibliography}

DOI http://dx.doi.org/ 10.1055/s-0032-1309383

Endoscopy 2012; 44: E240-E241

(c) Georg Thieme Verlag KG

Stuttgart · New York

ISSN 0013-726X

\section{Corresponding author}

\section{I.-L. Lee, MD}

Division of Gastroenterology Department of Internal Medicine Shuang Ho Hospital Taipei Medical University 291 Zhongzheng Road Zhonghe District

New Taipei City 23561

Taiwan

Fax: +886-2-22490088

liaowhgi@gmail.com 